Clio. Femmes, Genre, Histoire

$19 \mid 2004$

Femmes et images

\title{
Témoigner, résister : photographies de femmes au Chiapas
}

\section{Rocío N. Martínez}

\section{(2) OpenEdition \\ 1 Journals}

Édition électronique

URL : http://journals.openedition.org/clio/647

DOI : 10.4000/clio.647

ISSN : 1777-5299

Éditeur

Belin

Édition imprimée

Date de publication : 1 avril 2004

ISSN : 1252-7017

Référence électronique

Rocío N. Martínez, "Témoigner, résister : photographies de femmes au Chiapas », Clio. Femmes, Genre, Histoire [En ligne], 19 | 2004, mis en ligne le 23 août 2013, consulté le 01 mai 2019. URL : http:// journals.openedition.org/clio/647; DOI : 10.4000/clio.647

Ce document a été généré automatiquement le 1 mai 2019.

Tous droits réservés 


\title{
Témoigner, résister : photographies de femmes au Chiapas
}

\author{
Rocío N. Martínez
}

1 Les photographies que l'on présente ici relèvent d'objectifs distincts, même si elles se réfèrent à des formes similaires de lutte et de résistance des femmes indigènes du Chiapas.

2 Les trois premières photos montrent différents moments de la vie dans un campement de personnes déplacées de leurs villages d'origine, en raison de la "guerre invisible » qui sévit dans l'état le plus méridional du Mexique. Dans ce campement dénommé Actéal, appartenant au municipe de Chenalhó, dans la région de Los Altos du Chiapas, quarantecinq indigènes tsotsils, en majorité des femmes et des enfants, ont été assassinés le 22 décembre 1997, alors qu'ils priaient dans leur chapelle. Ils appartenaient tous à des familles de producteurs de café, membres de l'organisation «Las abejas » (les abeilles) qui, bien que se distinguant du mouvement zapatiste (notamment par le rejet de la lutte armée), partageait avec celui-ci de nombreuses revendications et s'opposait également au contrôle exercé par le Parti Révolutionnaire Institutionnel, alors au pouvoir. Les exécutants du massacre étaient membres de l'un des groupes paramilitaires formés sous l'égide de l'armée et du parti officiel, dans le cadre d'une stratégie contreinsurrectionnelle visant à semer la terreur non seulement parmi les communautés zapatistes mais aussi parmi celles qui refusaient de s'opposer à elles.

3 Le lendemain, des centaines de personnes de la société civile et de membres d'associations nationales et internationales se sont retrouvées sur les lieux du massacre, pour témoigner de l'horreur, face aux corps jetés dans des sacs en plastique: spectacle d'une réalité honteuse qui a bouleversé la conscience mexicaine. Dans cette situation douloureuse, le rôle des observateurs nationaux et internationaux fut déterminant pour réaliser des reportages susceptibles de faire connaitre au Mexique et dans le monde les témoignages des victimes et des communautés auxquelles elles appartenaient. Mais la majorité des reporters et des observateurs se retirèrent au fil des jours. Bien peu d'entre eux décidèrent de demeurer plus longuement, soit à Actéal, soit dans les autres 
campements installés pour tenter de garantir la sécurité des milliers de familles qui, en nombre croissant, durent abandonner leurs maisons, leurs animaux et leurs terres, sous la pression de la menace paramilitaire.

4 C'est dans ces circonstances que Bertrand Meunier, après avoir accompagné la communauté d'Actéal durant les jours de terreur extrême qui suivirent le massacre, fut comme «adopté » par elle et put assumer la responsabilité de capter avec son appareil photographique l'histoire alors vécue: la nécessité exprimée par la communauté consistait à témoigner ailleurs de « comment nous vivons et souffrons ici, mais aussi de comment nous luttons ». Le degré de confiance créé par une vie en commun partagée jour après jour (initialement durant une période de trois mois, puis lors de visites plus brèves répétées au cours des années 1998-2000) et le respect des normes internes du groupe expliquent que les photographies de Bertrand Meunier ne relèvent pas d'un reportage journalistique, qui soumet la réalité à ses rythmes fragmentés et rapides. Ses images se veulent un document poétique qui scrute lentement la profondeur du temps communautaire, dans lequel lui-même a été invité à s'immerger. Les clichés de femmes sélectionnés ici ne constituent donc pas un thème spécifiquement abordé en tant que tel par le photographe. Mais, parmi les histoires qu'il transmet, les images de femmes ont une place remarquable, en raison du rôle important que celles-ci assument dans les tâches matérielles et d'organisation de la vie du campement.

5 Contrastant nettement avec les trois premières, la quatrième image correspond à une pratique photographique différente. Il s'agit d'une photo diffusée par une célèbre agence internationale à l'intention des moyens de communication, lors de l'un des moments culminants de la très médiatisée Marche de la Dignité Indigène, en mars 2001 : la prise de parole, à la «plus haute tribune de la Nation» (le parlement mexicain), d'une femme indigène. La fonction de l'image est ici de rendre compte d'un événement spectaculaire, à la construction duquel le sujet photographié contribue avec la satisfaction affichée de se montrer sous un jour qui rompt avec l'image traditionnelle de la femme indigène, soumise à la victimisation de sa propre histoire comme femme et comme indigène.

Outre cette image, qui a fait le tour du monde, d'autres photos devenues emblématiques ont contribué à modifier l'image que les femmes se faisaient d'elles-mêmes dans les communautés et ont favorisé leur capacité à se reconnaître comme actrices de leur propre destin. Conscientes de l'utilisation et de la manipulation des images par les moyens de communication, de nombreuses femmes indigènes de différentes régions du Chiapas ont appris à se servir d'appareils photographiques et de caméras vidéo.

7 Ce sont elles désormais qui, en une vision qui leur est propre, expriment leur condition de femmes et la perception du monde qui les entoure. Dans certains cas, elles réfléchissent sur leur culture, à partir du regard porté sur les objets les plus simples, les animaux et les éléments naturels qui constituent leur cadre de vie. Dans d'autres cas, elles souhaitent avoir, au sein des communautés elles-mêmes, la maîtrise d'un instrument qui leur permet de témoigner des violations des droits humains, des incursions violentes de l'armée et des formes multiples d'intimidation auxquelles se heurtent les luttes des femmes et des communautés dans leur ensemble.

8 Ainsi, qu'il s'agisse ou non de moyens technologiques avancés, les images créées par les femmes (artisanat textile, broderie, poterie et objets d'usage quotidien) se modifient en même temps que les représentations qu'elles ont d'elles-mêmes. Le processus s'inscrit jusque dans les corps, par la transformation de leurs attitudes et de leurs gestes. Un imaginaire différent émerge de ces pratiques de lutte, par lesquelles les femmes prennent 
conscience de leur situation sociale, de leur appartenance de genre et de leur histoire comme indigènes.

Fig. 17 : Campement de déplacés, Janvier 1998

9 Quelques jours après le massacre d'Actéal, plusieurs centaines de familles, obligées de fuir leurs terres d'origine, se sont réunies dans différents campements, afin de rester en vie et de se protéger des groupes paramilitaires.

Conformément à la coutume, les femmes ont la charge de la préparation des aliments. Même dans les situations les plus difficiles, on n'abandonne pas les tâches quotidiennes. On fait les tortillas de maïs, qui sont le produit final d'un travail familial dans les champs. Très tôt, les petites filles apprennent de leurs mères que ce processus est une part indispensable de la survie. Avec elles, elles s'habituent à participer à la récolte, à égrener les épis, à moudre les grains (soit avec le metate, petite meule de pierre, soit avec un moulin métallique), à les mêler à un peu de chaux, selon le procédé du nixtamal qui les rend digestes, à aplatir chaque tortilla avec la paume de la main et à la placer sur une plaque de fer posée sur le feu. La fabrication des tortillas, commencée vers cinq heures du matin, est un rituel quotidien qui confère aux femmes un rôle décisif dans l'autosuffisance alimentaire de la famille et de la communauté.

Ce sont les femmes déplacées qui ont la charge de nourrir les habitants du campement (entre six cents et huit cents), tandis que les hommes s'en vont durant la journée, pour chercher du travail ou pour tenter de sauver une partie de leurs animaux, de leurs récoltes ou de leurs maisons brûlées, avec l'espoir de récupérer un jour leurs terres. Ce sont aussi ces femmes qui ont la charge des enfants et des anciens, début et fin de la trajectoire de vie, si souvent métaphore du cycle agricole qui a donné lieu aux multiples mythes d'origine et de régénération des peuples mayas. Ainsi, la résistance pour la vie se construit à partir des gestes les plus quotidiens, sans oublier la peine des morts : « au début, nous pensions que nous avions tout perdu ; maintenant nous savons que nous avons la vie, et il faut en prendre soin» (témoignage d'une femme tsotsile déplacée, 1998).

Fig. 18 : Femmes déplacées, Janvier 1998

Deux femmes indigènes de Chenalhó, déplacées de leur communauté, coupent le bois avec une hache et une machete. La jeune femme du premier plan coupe les troncs les plus gros, utilisant pour cela le poids énorme de la hache. La femme âgée, au second plan, surveille les alentours et tient dans sa main la machette, instrument caractéristique du paysan, qui permet d'ouvrir le chemin entre les herbes, mais aussi de couper le petit bois.

De la montagne et de ses arbres, richesse de la "Terre Mère ", on tire le nécessaire pour la construction de la maison, pour allumer le feu afin de préparer les aliments et de se chauffer durant les soirs glacés et humides de l'hiver. Même dans ces conditions de subsistance déplorables, le vêtement est impeccable : le Huipil, blouse tissée à la main et brodée par les femmes elles-mêmes ; le Nagua, jupe de coton également tissée et teintée d'indigo dans les communautés.

Être femme, être indigène, être femme indigène avec dignité est fortement lié à la conscience de l'importance de la lutte. De la lutte pour affirmer la différence des sexes, alors que les femmes réalisent souvent les mêmes activités que les hommes. De la lutte pour la reconnaissance de la différence culturelle en tant qu'indigène. De la lutte pour le respect de l'intégrité comme personne et comme genre, à l'égard de leur propres maris 
d'abord et jusqu'à l'inscription dans la Constitution mexicaine des droits et des cultures indigènes.

Fig. 19 : Marche des femmes, 22 décembre 1999 Actéal a été une démonstration de la lutte conjointe des femmes indigènes zapatistes et non zapatistes. Deux ans après le massacre, les femmes traversèrent les montagnes pour réclamer que justice soit rendue aux victimes d'Actéal. Le visage couvert d'un passemontagne ou d'un foulard à la façon des zapatistes, les femmes de Chenalhó et des municipes voisins ont ainsi témoigné des avancées organisationnelles de la résistance des femmes. À leurs rôles traditionnels, s'ajoutent les fonctions qu'elles assument bien souvent comme membres de coopératives de femmes pour la production alimentaire ou artisanale, comme responsables communautaires de santé ou d'éducation, ainsi qu'à tous les niveaux, tant militaires que politiques, du mouvement zapatiste. La lutte des femmes zapatistes a commencé avant le 1er janvier 1994 : au cours de l'année précédente, le vote de la «Loi révolutionnaire des femmes», malgré les réticences de nombreux compagnons, a marqué, comme le rapporte le sous-commandant $\operatorname{Marcos}^{2}$, le véritable début du soulèvement zapatiste.

Fig. 20 : La Commandante Esther à la tribune du Congrès Fédéral, 28 mars 2001

retentissante " Marche de la Couleur de la Terre », menée par l'EZLN (Ejercito zapatista de liberación nacional) en février-mars 2001, est partie de San Cristóbal de Las Casas pour porter la parole des indigènes insurgés du 1er janvier 1994, à travers les voix de leurs commandants et commandantes. Parmi celles-ci, les interventions des commandantes Yolanda, Susana et Esther ont eu un grand impact. Dans tous leurs discours, elles ont exposé la triple oppression vécue en tant que femmes, en tant qu'indigènes et comme part la plus pauvre de la société. Elles ont fait voir le racisme et le mépris qu'elles subissent dans leurs propres maisons, dans leurs propres communautés, dans leur propre pays. Elles ont expliqué l'importance de la lutte zapatiste des femmes, à côté des hommes, pour obtenir la reconnaissance de leurs droits et de leurs cultures comme indigènes, c'est-à-dire du respect de leur différence au sein de l'unité mexicaine.

17 Le 28 mars 2001, à la tribune du Congrès, où tout le monde s'attendait à voir Marcos prendre la parole, la surprise a été créée par une femme en vêtement traditionnel, frêle silhouette à la voix d'abord hésitante au milieu du solennel decorum national : «Voilà que c'est moi qui suis ici, une femme indigène. Personne ne pourra se sentir agressé, humilié ou dépossédé par le fait que j'occupe cette tribune et y prenne la parole (...) Mon nom est Esther, mais en ce moment, cela n'importe pas. Je suis zapatiste, mais en ce moment, cela n'importe pas. Je suis indigène et je suis une femme et cela seul importe maintenant (...). Ma voix a demandé et demande la reconnaissance constitutionnelle de nos droits et de notre culture ».

Malgré le succès de la Marche et l'accès de la parole indigène au parlement, deux ans plus tard, les Accords de San Andrès, pourtant signés par le gouvernement en 1996, n'ont toujours pas trouvé leur traduction constitutionnelle et legislative réclamée par les zapatistes et les mouvements indigènes du Mexique. 


\section{En guise de conclusion}

19 Le 9 août 2003, considéré par l'ONU comme la journée internationale des peuples indigènes, l'ELZN a annoncé, lors d'un rassemblement massif à Oventic (lieu également appelé «Résistance et rébellion pour l'humanité»), que, face à la surdité de la classe politique qui a empêché la reconnaissance constitutionnelle du droit à l'autodétermination des peuples indigènes, la pratique de l'autonomie entrait en vigueur dans les cinq régions qu'il contrôlait. Cet acte signifia, entre autres choses, un processus de passation du pouvoir de décision, de l'armée zapatiste vers la nouvelle figure civile des «juntas de buen gobierno» (organes situés dans les centres zapatistes rebaptisés pour la circonstance Caracoles, c'est-à-dire "Coquillages ou Escargots", et faisant office de coordination régionale des municipes autonomes zapatistes), avec une priorité accordée à l'autogestion en matière de production, de santé et d'éducation.

20 Parmi les discours de la comandancia general zapatiste, trois furent prononcés par les commandantes Rosalinda, Fidelia et Esther, qui réaffirmèrent notamment les sanctions prévues à l'encontre de ceux qui introduisent de l'alcool dans les communautés. Cette décision, prise à la demande des femmes ${ }^{3}$, qui sont les principales victimes, avec les enfants, des effets violents provoqués par l'abus d'alcool, est considérée comme une avancée importante de leur lutte. Les trois commandantes, chacune avec la tonalité particulière correspondant à sa personnalité singulière, soulignèrent que la lutte des femmes est partie intégrante de la transformation sociale promue par l'EZLN et, dans chacun de leurs discours, invitèrent les femmes indigènes du Mexique à mettre en pratique, dans la construction de l'autonomie, leurs propres droits comme femmes.

21 "Le seul chemin pour atteindre ce dont nous avons besoin consiste à bien nous organiser, à fortifier notre résistance et nos municipes autonomes. Mais pour y parvenir il est nécessaire que nous participions tous, que tous nous y mettions du nôtre et que nous les femmes nous ne restions pas en arrière. Seulement ainsi notre lutte pourra triompher " (commandante Rosalinda).

22 "Nous allons imposer obligatoirement qu'on nous respecte comme femmes que nous sommes, même si [les hommes] doivent pour cela prendre leur petit air triste. Car il y a encore de nombreux endroits du Mexique où nous autres, les femmes, sommes maltraitées, dépréciées, exploitées, où ils disent que nous ne servons à rien, que nous ne valons rien, que nous n'avons aucun droit ; mais aujourd'hui cette réalité doit disparaître et ce que nous allons faire, c'est leur imposer l'obligation de nous respecter » (commandante Fidelia).

23 «Il n'est plus temps de nous taire et de nous humilier devant les hommes, ni de leur demander qu'ils nous fassent la faveur de nous respecter. Il est temps d'agir par nousmêmes et d'obliger les hommes à respecter nos droits. Car si nous ne le faisons pas, personne ne le fera pour nous. Ce qui nous reste à faire maintenant, c'est agir, hommes et femmes, pour construire et avancer dans notre autonomie...

ILS NE PEUVENT PAS NOUS RETIRER CE QUE NOUS SOMMES » (commandante Esther). 


\section{BIBLIOGRAPHIE}

La Marcha del Color de la Tierra, Comunicados, cartas y mensajes del Ejército Zapatista de Liberación Nacional, del 2 de diciembre del 2000 al 2 de abril del 2001, Mexico, Editorial Rizoma-Causa Ciudadana, 2001.

Autonomía Rebelde, La fiesta de los Caracoles, Comunicados y Palabras de los Comandantes-Planes, 2003, Mexico, Ediciones Pirata.

FALQUET France Jules, 1995, La Violencia cultural del sistema educativo, las mujeres indigenas victimas de la escuela, San Cristobal de Las Casas, Instituto de Asesoría Antropológica para la Región Maya A.C.

GARZA CALIGARIS Ana María, 2000, «El Movimiento de Mujeres en Chiapas, Haciendo Historia », Anuario de Estudios Indígenas VIII, San Cristobal de Las Casas,Universidad Autonoma de Chiapas, pp.110-133.

HERNÁNDEZ CASTILLO Rosalva Aída (ed.), 1998, La Otra palabra, mujeres y violencia en Chiapas, antes y después de Acteal, Mexico, Centro de Investigaciones Estudios Superiores en Antropología Social. LE BOT Yvon (dir.), 2002, Indiens du Mexique, Exposition du Parc de La Villette, Paris, Éditions Indigène.

LoVera Sara y PAlomo Nellys (ed.), 1997, Las Alzadas, Mexico, Centro de Información de La Mujer. A.C.

Le Visage des femmes de Chiapas, 2000, Médiathèque de Noisy-le-Sec.

MILLÁN Margara, 1996, « Las zapatistas del fin del milenio. Hacia políticas de autorrepresentación de las mujeres indígenas » en Chiapas, 3. Instituto de Investigaciones Económicas, UNAM, Mexico, Ediciones ERA, pp. 19-32

Rebeldía : Palabras de los Comandantes Zapatistas en Oventik, 1, n 10, Mexico, 2003.

ROJAS Rosa, 1995, ¿Chiapas y Las mujeres Qué?, Tome I et II, Mexico, Taller Editorial La Correa Feminista.

ROVIRA Guiomar, 1996, Mujeres de Maíz, la voz de las indígenas de Chiapas y la rebelión zapatista, Barcelona, Virus.

Tierra de Mujeres. Propuestas de las mujeres indígenas al Congreso Nacional Indígena, 1996, Mexico, K’inal Antsetik. A.C.

\section{NOTES}

1. Polhó est devenu le siège d'un Municipe Autonome, dans la région de Chenalhó, ayant accueilli le plus grand nombre de déplacés (environ 10 000), zapatistes ou membres d'organisations proches.

2. Journal La Jornada, le 30 janvier 1994.

3. Demande faite depuis mars 1996 dans «la ampliación de las leyes revolucionarias de las mujeres ". 


\section{RÉSUMÉS}

Cet article analyse quatre photographies qui révèlent différents modes de vie et de résistance combattante des femmes indigènes au Chiapas. On les voit ainsi s'organiser et transformer leur propre image. En même temps, elles construisent leur autonomie à la lumière du genre et à travers la conscience de leur statut social et de leur appartenance au peuple indigène de Mexico. «Personne ne peut nous prendre ce que nous sommes» (Commandante Esther, EZLN)

This paper analyses four photographs that reveal the different ways of life and of militant resistance of the indigenous women in Chiapas. We see them as they organize themselves and transform their own image. At the same time they are constructing their autonomy in terms of gender and through their consciousness of their social status and as part of the indigenous people of Mexico. « Nobody can take from us what we are » (Comandante Esther, EZLN)

\section{INDEX}

Mots-clés : Chiapas, femme indigène, lutte zapatiste, résistance, photographie

\section{AUTEUR}

\section{ROCÍO N. MARTÍNEZ}

Rocío N. MARTÍNEZ est professeure d'histoire de l'art à l'université autonome du Chiapas (Mexico). Elle a publié Totik, metik, kanal... a dos al'art à l'uni (CIACH, San Cristóbal de Las Casas, 1999), « El cuerpo la casa y el universo, la construcción del espacio en los ninos desplazados de Chenalhó ", en II Coloquio, Nuevos actores políticos y sociales (DEAS, INAH, UNAM, San Cristóbal de Las Casas, 2000) et « OKOT -BA, La danza como patrimonio intangible, entre el folklore y el ritual », Haceres y Saberes de Chiapas, II Encuentro sobre el Patrimonio cultural de Chiapas (Tuxtla, Gutierrez, 2002). 